\section{Hand rotweiß}

Ein 14-jähriges Mädchen wurde zur Abklärung von seit drei Jahren bestehenden intermittierenden Episoden von akuten Schmerzen in Händen und Füßen vorgestellt.

- Auslöser für die Beschwerden waren in den meisten Fällen körperliche Aktivität und/oder Aufenthalt im Wasser. Während der körperlichen Untersuchung ließ sich durch eine repetitive Bewegung der rechten Hand ein brennender Schmerz und eine akute Rötung auslösen (s. Abb.). Ein ähnliches Phänomen trat auf, wenn die Patientin die Hand in warmem Wasser badete.

Die Symptomatik und die Auslöser sind typisch für die sog. Erythromelalgie, bei der es durch eine Erhöhung der Hauttemperatur zu schmerzhaften Attacken und Rotverfärbungen sowie Schwellungen an Händen und Füßen kommt.

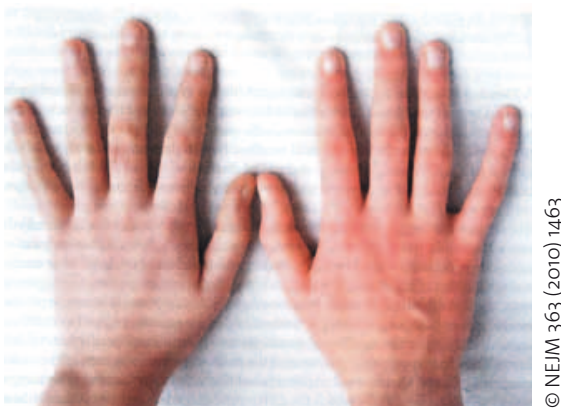

Rötung der rechten Hand nach Bewegung.

Abkühlung der betroffenen Extremität führt regelhaft zu einem Rückgang der Symptomatik. Der Symptomenkomplex ist assoziiert mit myeloproliferativen Erkrankungen, Kollagenosen, Vaskulitiden, Diabetes, Gicht, Multipler Sklerose, einer thrombozytopenischen Purpura und einer Reihe von Medikamenten.

Während die Symptome bei myeloproliferativen Erkrankungen üblicherweise gut auf Aspirin ansprechen, ist die Therapie der Symptome bei anderweitigen Grunderkrankungen schwierig. Man kann den Patienten nur raten, auslösende Faktoren möglichst zu meiden.

\section{Kommentar}

Selbst wenn die Behandlung wenig erfolgreich ist, so ist vielen Patienten doch damit gedient, wenn der behandelnde Arzt eine sichere Diagnose aufgrund der Anamnese und des klinischen Befundes stellt, den Patienten über die relative Harmlosigkeit aufklärt und Verhaltensmaßregeln an die Hand gibt.

H. S. FÜEßL

\footnotetext{
-D. Lipsker

A white hand and a red hand - erythromelalgia. New Engl. J. Med. 363 (2010) 15, 1463
} 